\title{
Strangulation of the umbilical cord by an amnion band - a rare cause of intrauterine demise: a case report Konstantinos Chatzigeorgiou*1, Theodoros Theodoridis', Ioannis Efstratiou ${ }^{2}$, Apostolos Athanasiadis ${ }^{1}$, Leonidas Zepiridis ${ }^{1}$, Filippos Tzevelekis ${ }^{1}$ and Basil Tarlatzis ${ }^{1}$
}

\author{
Address: ${ }^{1} 1$ st Department of Obstetrics and Gynecology, Aristotle University of Thessaloniki, Papageorgiou General Hospital, Ring Road, Nea \\ Efkarpia, 56403 Thessaloniki, Greece and 2Department of Pathology, Papageorgiou General Hospital, Ring Road, Nea Efkarpia, 56403 \\ Thessaloniki, Greece \\ Email: Konstantinos Chatzigeorgiou* - chatzkon@hotmail.com; Theodoros Theodoridis - theodtheo@yahoo.gr; \\ Ioannis Efstratiou - dr.efstratiou@gmail.com; Apostolos Athanasiadis - apostolosa@hol.gr; Leonidas Zepiridis - lzepi@hol.gr; \\ Filippos Tzevelekis - ftzev@med.auth.gr; Basil Tarlatzis - tarlatzis@hol.gr \\ * Corresponding author
}

Published: 29 November 2009

Cases Journal 2009, 2:9108 doi:10.1186/1757-1626-2-9108

This article is available from: http://www.casesjournal.com/content/2/1/9108

(C) 2009 Chatzigeorgiou et al; licensee BioMed Central Ltd.

This is an Open Access article distributed under the terms of the Creative Commons Attribution License (http://creativecommons.org/licenses/by/2.0), which permits unrestricted use, distribution, and reproduction in any medium, provided the original work is properly cited.

\begin{abstract}
Introduction: The amniotic band syndrome has a scarce prevalence and intrauterine death as a result of amniotic bands formation is extremely rare.

Case presentation: We present an illustrative case of intrauterine death of an embryo in the 24th gestational week in an 30-year old primigravida. The death was ascribed to the twisting of the umbilical cord around the left upper extremity, causing a strangulation of the umbilical cord in a very impressive way.
\end{abstract}

Conclusion: Constriction of the umbilical cord by an amniotic band is extremely rare and very hard, if not impossible, to diagnose with antenatal sonography.

\section{Background}

The amniotic band syndrome, as a cause of fetal malformations, has a prevalence ranging from one in 1200 up to one in 15000 live births, and only rare cases of intrauterine fetal death by constriction of the umbilical cord have been described $[1,2]$. The aetiology of band formation remains unknown.

We present an illustrative case of intrauterine death of an embryo in the 24th gestational week due to constriction of the umbilical cord by an amniotic band.

\section{Case presentation}

A 30-year-old Caucasian primigravida of Greek nationality, presented at the 18th pregnancy week. She had up to this time a fragmentary antenatal care, without first trimester sonography and without serologic evaluation for TORCH. Her medical history however, was unremarkable, without prior surgeries. During the first visit, sonographic evaluation confirmed the presence of a singleton male fetus, without obvious anatomical deformities, and fetal biometry corresponding to the 18th gestational week. She was referred for detailed fetal anatomy scan in the 23th 
gestational week. During this detailed scan, again no obvious deformities could be identified, but due to the position of the embryo, the heart and the upper extremities could not clearly be seen. Therefore, the patient was rescheduled for a second visit one week later. Unfortunately, in this second visit, only intrauterine demise was ascertained, in the $23+4$ week.

Termination of pregnancy through induction of labour took place on the next day with vaginal $600 \mathrm{mcg}$ misoprostol. A $600 \mathrm{~g}$ stillborn, male embryo was delivered, with a CRL of $22 \mathrm{~cm}$. In approximately $12 \mathrm{~cm}$ from the placental insertion, the umbilical cord was twisted around the left upper extremity, causing a strangulation of the umbilical cord in a very impressive way (Figure 1). The cord inserted at the left hand which was hypoplastic and severely deformed (Figure 2). No other abnormalities could be found on autopsy. The placenta weighted $208 \mathrm{~g}$ and measured $14 \times 9 \times 2 \mathrm{~cm}$, while the umbilical cord measured $30 \mathrm{~cm}$ and his architecture was normal with three vessels.

\section{Discussion}

Many clinical deformities in the meaning of amniotic bands have been encountered and range from simple ring constrictions to major craniofacial and visceral defects [3]. Very often, where a band is attached to the fetus there is usually a local deformity, as described in our case [4]. However, only rare cases of intrauterine fetal death by constriction of the umbilical cord have been described in the literature, mostly in the second and third trimester $[5,6]$ while the first description in modern literature was published in 1953, by Craven and Geddes [7].

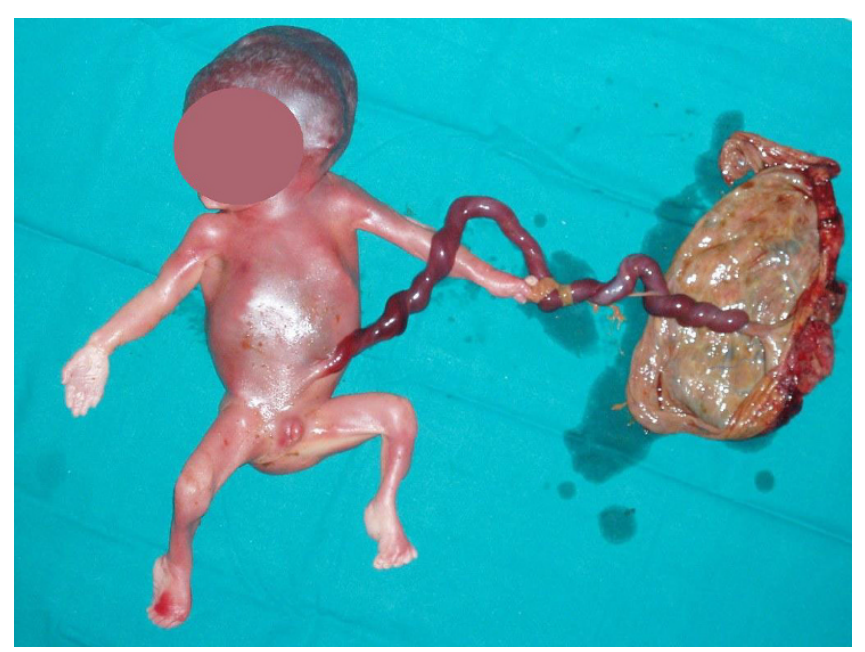

Figure I

Twisting of the umbilical cord around the left upper extremity, causing a strangulation of the umbilical cord.

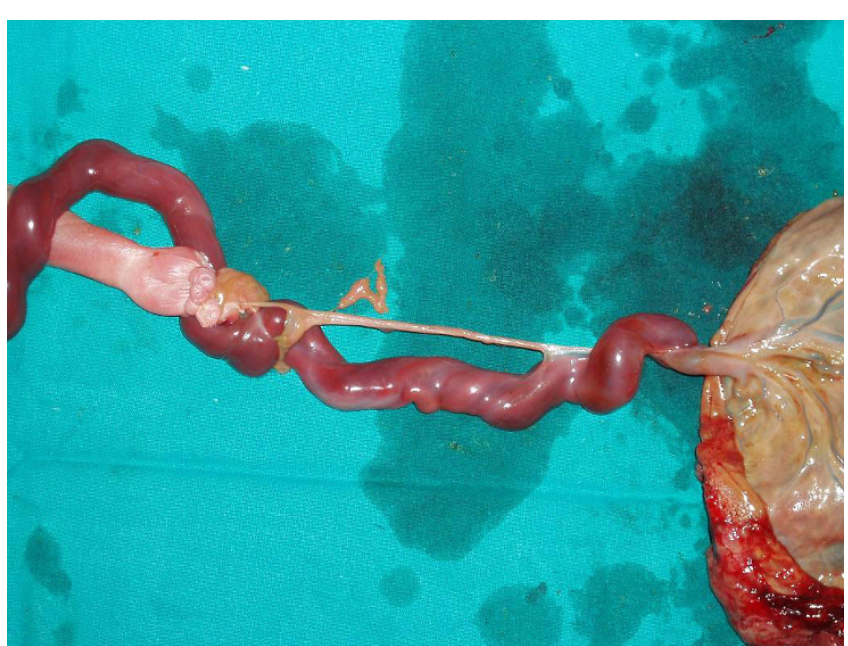

Figure 2

Insertion of the umbilical cord at the hypoplastic and severely deformed left hand.

The aetiology of the formation of amniotic bands remains controversial. The most accepted hypothesis is that of Torpin, who suggested that early amnion rupture might be the precipitating event. The amnion is torn during the first trimester and this abnormal amnion might form bands with the chorionic mesenchyma which can attach to the embryo [8].

Early diagnosis is usually not possible and might be suspected only in presence of other embryonic malformations. Elevation of $\beta$-HCG as a result of placental attempt to counteract the fetal growth restriction and hypoxia, due to the strangulation of umbilical cord by the amniotic bands, has also be described [3]. However, the practical aspect of determination of $\beta$-HCG in advanced pregnancy is questionable.

\section{Conclusion}

Constriction of the umbilical cord by an amniotic band leading to intrauterine fetal death is extremely rare and very hard, if not impossible, to diagnose with antenatal sonography.

\section{Abbreviations}

TORCH: Toxoplasmosis, Other Agents, Rubella, Cytomegalovirus, Herpes Simplex; $\beta$-HCG: beta - human chorionic gonadotropin.

\section{Consent}

Written informed consent was obtained from the patient for publication of this case report and accompanying images. A copy of the written consent is available for review by the Editor-in-Chief of this journal. 


\section{Competing interests}

The authors declare that they have no competing interests.

\section{Authors' contributions}

KC and TT participated in patient management, acquisition of data, interpretation of data, and were the major contributors in writing the manuscript. IE performed the pathology of the specimen. AA participated in patient management. LZ, FT and BT critically revised the paper. All authors read and approved the final manuscript.

\section{References}

I. Higginbottom MC, Jones KL, Hall BD, Smith DW: The Amniotic band disruption complex: Timing of amniotic rupture and variable spectra of consequent defects. J Pediatr 1979, 95:544-549.

2. Burton DJ, Filly RA: Sonographic Diagnosis of the Amniotic Band Syndrome. AJR I991, I56:555-558.

3. Sifakis S, Mantas N, Konstantinidou A, Koukoura O, Avgoustinakis E, Koumantakis E: A stillborn fetus with amniotic band syndrome and elevated levels of alpha-fetoprotein plus beta-human chorionic gonadotropin: a case report. Fetal Diagn Ther 2008, 24(2): III-II4.

4. Rushton DI: Amniotic band syndrome. BMJ 1983, 286:919-920.

5. Reles A, Friedmann W, Vogel M, Dudenhausen JW: Intrauterine fetal death after strangulation of the umbilical cord by amniotic bands. Geburtshilfe Frauenheilkd I991, 5 I(12): 1006-1008.

6. Lurie S, Feinstein M, Mamet Y: Umbilical cord strangulation by an amniotic band resulting in a stillbirth. J Obstet Gynaecol Res 2008, 34(2):255-257.

7. Craven FO, Geddes C): Neonatal Death due to Ligation of Umbilical Cord by an Amniotic Band. BMJ 1953, I (4801):81.

8. Torpin R: Fetal malformations caused by amnion rupture during gestation Ist edition. Springfield (IL): Charles C Thomas Publisher; 1963:3-53.

Publish with Bio Med Central and every scientist can read your work free of charge

"BioMed Central will be the most significant development for disseminating the results of biomedical research in our lifetime. "

Sir Paul Nurse, Cancer Research UK

Your research papers will be:

- available free of charge to the entire biomedical community

- peer reviewed and published immediately upon acceptance

- cited in PubMed and archived on PubMed Central

- yours - you keep the copyright 